\title{
LETTER
}

doi:10.1017/S104161021000178X

\section{Serum vitamin B12, folic acid, and hemoglobin and cognition in Alzheimer's disease}

Alzheimer's disease (AD) is a heterogeneous disorder that has a multifactorial etiology. No single factor can account for all cases by itself. Dietary surveys have shown that the elderly often have low intakes of vitamins and that their plasma or red blood cell folate levels are often low. Low blood levels of other nutrients like vitamin $\mathrm{C}$, thiamine and 25-hydroxycholecalciferol have also been found to be common.

Diet, dietary fat and caloric intake have been shown to be important risk factors for $\mathrm{AD}$ in several countries with fish consumption being found to be a protective factor (Grant et al., 2002). There are limited data on the direct association between dietary intake of folic acid and vitamin $\mathrm{B} 12$ and incidence of AD. Some clinical trials have found vitamin supplements to have no effect on cognition among older persons. For example, Corrada et al. (2005) found an inverse correlation with folate intake but no association with intake of vitamins B12.

The aim of our study was to test the correlation between vitamin B12, folate, and hemoglobin and cognition in late-onset $\mathrm{AD}$. The study was approved by the Local Research Ethics Committee and Local Research and Development Department.

All new referrals $(n=74)$ to a Memory Clinic with a clinical diagnosis of late-onset $\mathrm{AD}$ were studied over a period of six months. Cognition was assessed using the Mini-mental State Examination (MMSE) and/or Addenbrooke's Cognitive Examination Revised (ACE-R). Fasting blood samples including hemoglobin, serum folate levels and serum vitamin B12 levels were collected in the Memory Clinic and analyzed in the Pathology Department of the hospital. ACE-R/MMSE scores and blood investigation results were analyzed for possible correlation.

A total of 74 participants were involved in the study. Their mean age was 80.1 years (SD 7.65), and $35.1 \%$ were male and $64.9 \%$ were female. Their mean MMSE score was 20.82 points (SD 4.44) and the mean ACE-R score was 65.8 points (SD 12.03). Serum vitamin B12 had a very wide range of $60 \mathrm{ng} / \mathrm{L}$ to $2000 \mathrm{ng} / \mathrm{L}$, giving a mean of $390.92 \mathrm{ng} / \mathrm{L}$ (SD 350.26) while mean serum folate was $6.95 \mu \mathrm{g} / \mathrm{L}$
(SD 4.95). Serum hemoglobin results had a range of $10.5 \mathrm{~g} / \mathrm{dL}$ to $18.4 \mathrm{~g} / \mathrm{dL}$ with a mean of $13.67 \mathrm{~g} / \mathrm{dL}$ (SD 1.43).

There was a significant correlation between the MMSE scores and the ACE- $\mathrm{R}$ (Pearsons $\mathrm{r}=0.775$, $p<0.001$ ) but did not correlate with B12, folate or hemoglobin levels $(p>0.05)$. Vitamin B12 and hemoglobin levels were found to have a negative correlation (Pearsons $r=-0.266, p<0.05$ ). Vitamin B12 correlated with folate levels in the male subgroup $(\rho=0.536, p<0.05)$. ANOVA showed that MMSE and ACE-R have no significant effect with age, gender, $\mathrm{B} 12$, and $\mathrm{Hb}(p>0.05)$. However, ANOVA showed that ACE-R only has a significant effect with serum folate, $F(14,3)=$ $21.537, p<0.01$.

These data are cross-sectional in nature and should not be interpreted as causal. Serum homocysteine and ferritin levels are not measured routinely in clinical practice. This has prevented the testing of the relationship between these variables and B12 and folic acid levels.

In this study we sought to reflect the routine clinical setting and ensure that the physical needs of our elderly patients would be met, especially given that deficiencies are easy to treat once discovered. This has affected the sample size as we excluded any other diagnoses, mixed diagnoses and any clients with vascular risk factors or who were taking vitamins supplements as potential confounders.

The findings of our study agree with those of Kim et al. (2008) who showed that folate levels correlated positively with vitamin B12 levels. However, this correlation appeared to hold true among male participants only. The mean hemoglobin level and serum vitamin B12 levels were similar to the findings of Engelborghs et al. (2004).

Several socio-medical problems have direct impact on the nutritional status of the elderly. The decline of extended family networks in developed societies means that the elderly potentially suffer loneliness, lack of support, poor social stimulation and even neglect by their family, at an age when they are likely to have few other means of sustaining their needs. The presence of crippling physical ill-health and sensory impairments would also compound their problems. It is therefore a great compliment to the health and social service structure in the U.K. that cases of gross neglect and malnutrition among the aged is not as common as might be expected.

Although other workers have reported abnormal levels of vitamin B12, folate and/or hemoglobin in 
people with dementia, it remains unclear whether this preceded the dementia or resulted from it, just as it has not been clear whether dementia results in poor dietary intake or conversely, whether declining dietary intake of the elderly contributes to the dementing process.

The question that therefore remains to be answered is whether there is a case for routine assays of vitamin B12, folate and hemoglobin in patients being screened for dementing illnesses like $\mathrm{AD}$. High dietary intake of folate and vitamin B12 or supplements may be protective against cardiovascular disease, stroke and dementia (Luchsinger et al., 2007). Blood folate on the other hand has been found to correlate negatively with symptoms of cognitive function in a mixed group of patients with dementia (Nilsson et al., 2000). Malouf and Areosa Sastre (2009) found no compelling evidence for any impact of vitamin B12 therapy on the cognitive function of elderly subjects with a vitamin B12 deficiency nor on those with a clinical diagnosis of dementia. Also, vitamin B12 deficiency contributed $1 \%$ to reversible cases of dementia. It is our opinion that routine blood investigations - including full blood count, vitamin B12 and folate levels - would serve as general health markers and that deficiencies when found would then be very easily treatable.

In this study, we found no correlations between levels of vitamin B12, folate and hemoglobin with cognition. However, these tests are helpful in assessing the general well-being of patients who, being elderly, have increased susceptibility to physical ill-health.

\section{References}

Corrada, M. M., Kawa, C. H., Hallfrisch, J., Muller, D. and Brookmeyer, R. (2005). Reduced risk of Alzheimer's disease with high folate intake: the Baltimore Longitudinal Study of Aging, Alzheimer's and Dementia, 1, 11-18.

Engelborghs, S. et al. (2004). Correlations between cognitive, behavioural and psychological findings and levels of vitamin B12 and folate in patients with dementia. International Fournal of Geriatric Psychiatry, 19, 365-370.

Grant, W. B., Campell, A., Itzhaki, R. F. and Savory, J. (2002). The significance of environmental factors in the etiology of Alzheimer's disease. Fournal of Alzheimer's Disease, 4, 179-189.

Kim, J. M. et al. (2008). Changes in folate, vitamin B12 and homocysteine associated with incident dementia. Fournal of Neurology, Neurosurgery and Psychiatry, 79, 864-868.

Luchsinger, J. A., Tang, M., Miller, J., Green, R. and Mayeux, R. (2007). Relation of higher folate intake to lower risk of Alzheimer's disease in the elderly. Archive of Neurology, 64, 86-92.

Malouf, R. and Areosa Sastre, A. (2009). Vitamin B12 for cognition. Cochrane Database of Systematic Reviews.

Nilsson, K., Gustafson, L. and Hultberg, B. (2000). The plasma homocysteine concentration is better than that of serum methylmalonic acid as a marker for sociopsychological performance in a psychogeriatric population. Clinical Chemistry, 46, 691-696.

V. M. AZIZ AND O. IsAAC

Email: victoraziz@doctors.org.uk 